\title{
Oral corticosteroid prescription patterns for asthma in France, Germany, Italy and the UK
}

\author{
Trung N. Tran ${ }^{1}$, Elizabeth King ${ }^{2}$, Rajiv Sarkar ${ }^{3}$, Cassandra Nan $^{4}$, \\ Annalisa Rubino ${ }^{5}$, Caroline 0’Leary ${ }^{2}$, Ruvimbo Muzwidzwa², Laura Belton ${ }^{6}$ \\ and Jennifer K. Quint ${ }^{7}$
}

Affiliations: ${ }^{1}$ AstraZeneca, Gaithersburg, MD, USA. ${ }^{2} \mathrm{Q} Q \mathrm{VIA}$, London, UK. ${ }^{3} \mathrm{IQVIA}$, Bengaluru, India. ${ }^{4}$ AstraZeneca, Mölndal, Sweden. ${ }^{5}$ Evidera, London, UK. ${ }^{6}$ AstraZeneca, Cambridge, UK. ${ }^{7}$ National Heart and Lung Institute, Imperial College London, London, UK.

Correspondence: Trung N. Tran, AstraZeneca, Gaithersburg, MD, USA. E-mail: trung.tran1dastrazeneca.com

@ERSpublications

This study gives a real-world snapshot of oral corticosteroid (OCS) use in western Europe, by highlighting an opportunity to shift towards corticosteroid-sparing therapies or safer alternatives that mitigate the risk of OCS-associated adverse effects http://bit.ly/3cB8kk8

Cite this article as: Tran TN, King E, Sarkar R, et al. Oral corticosteroid prescription patterns for asthma in France, Germany, Italy and the UK. Eur Respir J 2020; 55: 1902363 [https://doi.org/10.1183/ 13993003.02363-2019].

ABSTRACT Oral corticosteroids (OCS) are used to manage asthma exacerbations and severe, uncontrolled asthma, but OCS use is associated with adverse effects. We aimed to describe the patterns of OCS use in the real-world management of patients with asthma in western Europe.

We used electronic medical records from databases in France, Germany, Italy and the United Kingdom from July 2011 through February 2018. Patients aged $\geqslant 12$ years with an asthma diagnosis, at least one non-OCS asthma medication within \pm 6 months of diagnosis, and available data $\geqslant 6$ months prior to and $\geqslant 90$ days after cohort entry were included. High OCS use was defined as OCS $\geqslant 450 \mathrm{mg}$ prescribed in a 90-day window during follow-up. Baseline characteristics and OCS use during follow-up were described overall and by OCS use status.

Of 702685 patients with asthma, 14-44\% were OCS users and 6-9\% were high OCS users at some point during follow-up. Annual prevalence of high OCS use across all countries was $~ 3 \%$. High OCS users had a mean of between one and three annual OCS prescriptions, with an average daily OCS dosage of 1.3$2.2 \mathrm{mg}$. For patients who continued to meet the high-use definition, daily OCS exposure was generally stable at $5.5-7.5 \mathrm{mg}$ for $\geqslant 2$ years, increasing the risk of adverse effects.

Our study demonstrates that OCS use is relatively common across the four studied European countries. Data from this study may provide decisive clinical insights to inform primary care physicians and specialists involved in the management of severe, uncontrolled asthma.

Data underlying the findings described in this manuscript may be requested in accordance with AstraZeneca's data-sharing policy described at https://astrazenecagroup-dt.pharmacm.com/DT/Home

This article has supplementary material available from erj.ersjournals.com

Received: 9 Dec 2019 | Accepted after revision: 27 Feb 2020

Copyright OERS 2020. This version is distributed under the terms of the Creative Commons Attribution NonCommercial Licence 4.0. 


\section{Introduction}

Asthma is a chronic respiratory disease affecting an estimated 339 million people worldwide [1]. Prevalence is high across western and northern European countries [2], with studies over the past two decades reporting estimated asthma prevalence of $11-18 \%$ in the United Kingdom (UK), 10\% in France, $6.3-10 \%$ in Germany and $6-11 \%$ in Italy [3-8].

National and international treatment guidelines recommend a stepwise approach to asthma therapy, aimed at optimising symptom control and reducing risk of exacerbations [9, 10]. Despite these guideline recommendations, patients with asthma remain at risk of severe exacerbations because of lack of adherence to maintenance therapy, overuse of reliever therapy, poor inhaler technique, comorbidities or difficult-to-treat asthma. Asthma exacerbations are commonly treated with short bursts of oral corticosteroids (OCS), with OCS treatment overall associated with increased risk of adverse effects, ranging from acute complications such as infections to chronic complications such as metabolic and cardiovascular events [11-14]. In addition, recent cohort studies have demonstrated a dose-response relationship between OCS and adverse effects [14, 15], with the risk of some systemic adverse effects becoming statistically significant at cumulative exposures of $0.5-<1 \mathrm{~g}$, the equivalent of four lifetime OCS courses [15].

Until recently, the Global Initiative for Asthma (GINA) recommended OCS as add-on therapy for patients whose asthma remained uncontrolled despite receiving the highest possible inhaled corticosteroid (ICS) dosage. However, based on increasing evidence of OCS-related adverse effects and the availability of OCS-sparing biologic therapies, these recommendations have been updated to reflect that OCS add-on therapy should be considered carefully [9].

Despite increasing evidence and awareness of OCS-related adverse effects in general, data on OCS use patterns in European countries are limited, although country-specific evidence is essential to facilitate successful implementation of updated treatment recommendations in clinical practice. The current study aimed to describe OCS use patterns as well as demographics and clinical characteristics of patients with asthma prescribed OCS in France, Germany, Italy and the UK.

\section{Materials and methods}

\section{Data source}

This was a multicountry retrospective cohort study that used data from the following IQVIA electronic medical record databases from July 1, 2011 to February 28, 2018: IQVIA Medical Research Data (IMRD) incorporating The Health Improvement Network (THIN, a Cegedim database) in the UK [16, 17], Disease Analyzer in Germany [18], and Longitudinal Patient Data (LPD) in France [19] and Italy [20]. All data sources were carefully selected to contain nationally representative primary care data on patient demographics, diagnoses and medications, including prescription date and dosage. THIN includes data from $>3.1$ million active patients, representing nearly $5 \%$ of the UK population. Data are generally representative of the UK for age and sex comparisons, and quality outcome framework for chronic disease prevalence $[16,17]$. The German Disease Analyzer is based on patient records continuously collected from 2500 computerised practices ( $\sim 3 \%$ of all primary care physicians), providing information for $>11$ million patients throughout Germany [18]. In addition, information on hospital admissions was recorded in IMRD (UK), and pulmonologist care data were available for Germany. Hospital admission information was included to capture the wider healthcare resource implications of severe asthma. For Germany, pulmonologist care and general physician data were analysed separately, because the patients from these two panels were not mutually exclusive. The French and Italian LPDs collect medical information from proprietary practice management software used by physicians during patient office visits to record daily patient interactions, which, therefore, reflect routine clinical practice in the country. The panel of contributing physicians is maintained as a representative sample of the primary care physician population based on age, sex and geographical distribution, all of which are known to influence prescribing [19, 20].

\section{Study population}

Patients with active asthma (asthma diagnosis during the study period and at least one non-OCS asthma medication within \pm 6 months of diagnosis) aged $\geqslant 12$ years were included if data were available during $\geqslant 6$ months prior to index date (baseline period) and $\geqslant 90$ days after the index date. The index date (i.e. cohort entry date) was the day after the latest of 1) asthma diagnosis during the study period; 2) availability of $\geqslant 6$ months of data within the study period; or 3) non-OCS asthma medication within the study period (and recorded within \pm 6 months of a qualifying asthma diagnosis) (figure 1). Patients were followed until the first of the following events: end of the study period, loss to follow-up, or death.

Patients were excluded from all analyses if they had other respiratory conditions (COPD, lung cancer, idiopathic pulmonary fibrosis, pulmonary artery hypertension or cystic fibrosis), or conditions probably 


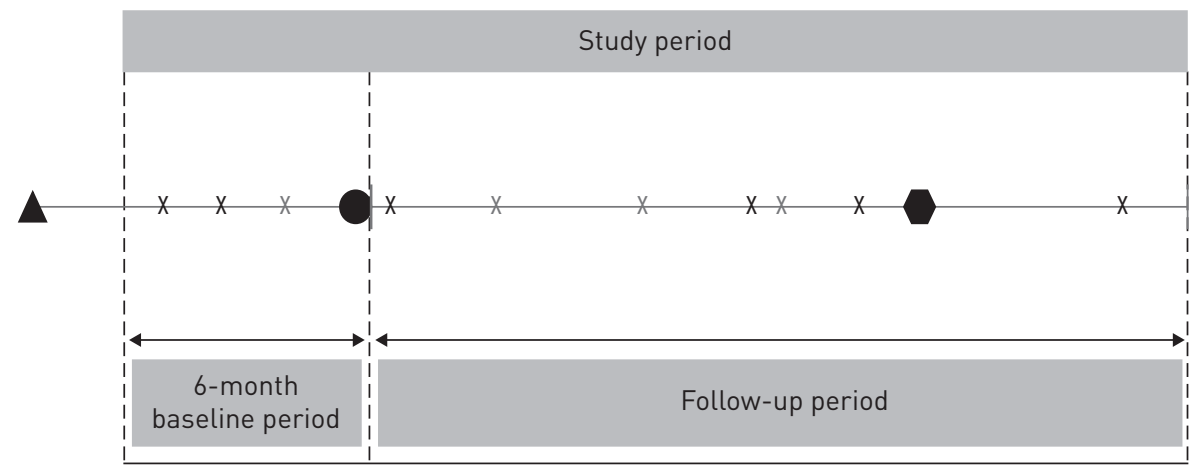

July 1, 2011

Index date\#

End of study period?
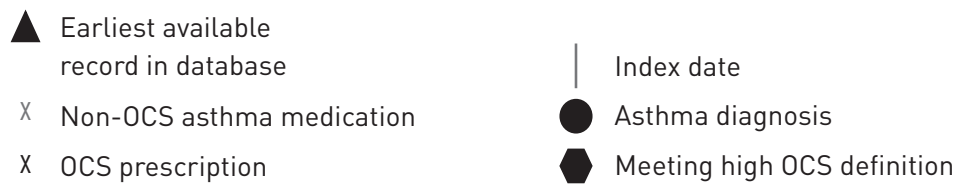

FIGURE 1 Illustration of the study period. OCS: oral corticosteroids. * : the day after the latest of an asthma

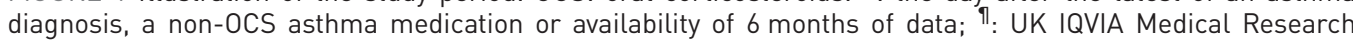
Data: January 17, 2018; Germany Disease Analyzer and France Longitudinal Patient Data (LPD): February 28, 2018; Italy LPD: December 31, 2017.

requiring OCS (inflammatory bowel disease, rheumatoid arthritis or systemic lupus erythematosus) at any time in their medical histories.

\section{Patient demographics and clinical variables}

Patient age, sex, body mass index (BMI; adults only) and smoking history were described at study index date. In addition, asthma medications (per Gemscript in UK and Anatomical Therapeutic Chemical classification in other countries) during the baseline period and comorbid conditions recorded at any time in patients' medical histories (per International Classification of Diseases (ICD)-9 (Italy), ICD-10 (Germany, France), and Read codes (UK)) were described. Asthma treatment step during the baseline period was obtained through an algorithm based on the GINA 2018 recommendations [1]. Asthma severity was categorised as mild (GINA steps 1-2), moderate (GINA step 3) and severe (GINA steps 4-5), or nonsevere (GINA steps 1-3) and severe (GINA steps 4-5). Over the baseline period, an exacerbation was defined as a single OCS prescription with total dosage $\leqslant 300 \mathrm{mg}$ or duration $\leqslant 10$ days. However, in the UK, OCS prescriptions with total dosage $\leqslant 300 \mathrm{mg}$ prescribed during an annual asthma review were not considered in this definition, as we assumed these prescriptions were to be used on an as-needed basis. In addition, prescriptions meeting the exacerbation definition recorded within 14 days of each other were considered part of the same exacerbation event.

\section{OCS exposure}

The annual number of OCS prescriptions and average daily dosage were described through all available data during the follow-up period (post-index date). Patients were classified as high OCS users, low OCS users and non-OCS users based on their prednisone-equivalent dosages. High OCS use was defined as a cumulative dosage $\geqslant 450 \mathrm{mg}$ within 90 days, corresponding to an average daily OCS dosage $\geqslant 5 \mathrm{mg}$ (supplementary figure S1). A dose-response relationship between average daily or cumulative OCS dosages and OCS-related complications has been reported, suggesting that these measures can be used to track the burden of high OCS use $[13,15,21-23]$. Patients who were prescribed OCS but did not meet the high OCS criteria were classified as low OCS users. Non-OCS users were those who had no OCS prescriptions during the entire follow-up period.

\section{Statistical analyses}

Data were analysed descriptively with a complete-case approach, whereby patients with missing data for relevant variables were excluded. Analyses were stratified by OCS use (high, low, no use), asthma severity (non-severe (GINA steps 1-3), severe (GINA steps 4-5)) and baseline exacerbations (presence, absence). Annual prevalence of high OCS use was calculated as the percentage of patients at the beginning of each calendar year who met the high OCS use definition during each calendar year. Because patients had to have data available for $\geqslant 6$ months before index date, annual prevalence of high OCS use was calculated 
only for 2012-2017 to allow for a full year of follow-up data for most patients. Average daily dosage for patients who continued to meet the high OCS use definition was calculated for each 90-day period for up to 2 years after the patient first met the definition. All analyses were performed via SAS (version 9.4; SAS Institute, Cary, NC, USA).

\section{Results \\ Study population}

Across the four countries studied, a total of 702685 patients met the study inclusion and exclusion criteria (figure 2). Median duration of follow-up was 33-55 months for all countries. Table 1 describes baseline characteristics of the study population per country. Mean age at index date across the countries was $42-$ 48 years, $57-63 \%$ of patients were female and mean BMI was $26.8-28.2 \mathrm{~kg} \cdot \mathrm{m}^{-2}$. The majority of patients (71-97\%) did not have an exacerbation during the 6-month baseline period. Up to $27 \%$ of patients were prescribed short-acting $\beta_{2}$-agonists only, while $58-80 \%$ of patients were prescribed at least an ICS inhaler during baseline. $\sim 40-50 \%$ of study populations across countries were categorised as having mild asthma (GINA steps 1-2), while $17-43 \%$ were categorised as having severe asthma (GINA steps 4-5). Comorbidity profiles of included patients are presented in table 1 .

\section{OCS exposure}

Across the four countries studied, $14-44 \%$ of patients had an OCS prescription and 6-9\% were classified as high OCS users at some point during follow-up (figure 3). Annual prevalence of high OCS use remained stable, at $\sim 3 \%$ across all countries during 2012-2017 (figure 4). Prescription patterns and frequency of OCS use stratified by treatment intensity are presented in table 2 . The average number of annual OCS prescriptions during follow-up was one to three for high OCS users and 0.5-0.6 for low users. The percentages of high and low OCS users receiving at least one OCS prescription per year were 33-72\% and $11-18 \%$, respectively. The corresponding ranges for four or more OCS prescriptions per year were 4$21 \%$ and $0.3-0.6 \%$ for high and low OCS users, respectively. On average, during the entire follow-up period, including the period when patients were not high OCS users, patients with high OCS use were exposed to an average daily OCS dosage from $1.3 \mathrm{mg}$ (Italy) to $2.2 \mathrm{mg}$ (UK). In contrast, patients with low OCS use had an average daily dosage from 0.2 to $0.3 \mathrm{mg}$.

During the 90-day period in which patients first met the high OCS use definition, average daily OCS dosage ranged from 7.2 to $10.3 \mathrm{mg}$ across the four study countries. Following a decrease to $\sim 2.5 \mathrm{mg} \cdot \mathrm{day}^{-1}$
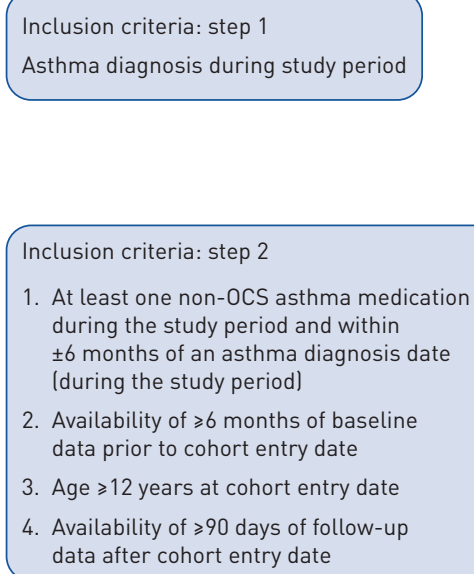
during the study period and within \pm 6 months of an asthma diagnosis date (during the study period)

2. Availability of $\geqslant 6$ months of baseline data prior to cohort entry date

3. Age $\geqslant 12$ years at cohort entry date

4. Availability of $\geqslant 90$ days of follow-up data after cohort entry date

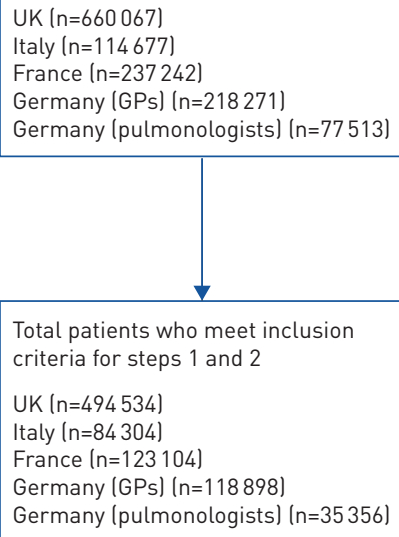

Total patients who meet inclusion

criteria for steps 1 and 2

UK ( $n=494534)$

Italy ( $n=84304)$

France $(n=123$ 104)

Germany (GPs) ( $n=118898$ )

Germany (pulmonologists) ( $n=35356$ )

Exclusion criteria

Diagnosis of\#:

1. COPD

2. Lung cancer

3. Idiopathic pulmonary fibrosis

4. Pulmonary artery hypertension

5. Cystic fibrosis

6. Inflammatory bowel disease

7. Rheumatoid arthritis

8. Systemic lupus erythematosus

FIGURE 2 Patient flowchart. UK: United Kingdom; GPs: general physicians; OCS: oral corticosteroids. \#: at any point in patient's medical record. 
TABLE 1 Demographics and baseline clinical characteristics of all included patients with asthma

\begin{tabular}{|c|c|c|c|c|c|}
\hline & \multicolumn{5}{|c|}{ Country } \\
\hline & UK & Italy & France & $\begin{array}{l}\text { Germany } \\
\text { (GPs) }\end{array}$ & $\begin{array}{c}\text { Germany } \\
\text { (pulmonologists) }\end{array}$ \\
\hline Age years & $42.4 \pm 19.1$ & $46.2 \pm 19.6$ & $42.7 \pm 19.6$ & $43.5 \pm 18.5$ & $47.5 \pm 17.7$ \\
\hline Female & 57.2 & 57.2 & 58.7 & 57.8 & 63.1 \\
\hline \multicolumn{6}{|l|}{ BMI $^{\#}$} \\
\hline$<18.5 \mathrm{~kg} \cdot \mathrm{m}^{-2}$ & 1.8 & 3.1 & 3.6 & 1.8 & 1.0 \\
\hline$\geqslant 18.5-<25 \mathrm{~kg} \cdot \mathrm{m}^{-2}$ & 33.1 & 38.0 & 37.3 & 31.9 & 31.7 \\
\hline$\geqslant 25-<30 \mathrm{~kg} \cdot \mathrm{m}^{-2}$ & 33.1 & 34.3 & 30.2 & 33.6 & 34.4 \\
\hline$\geqslant 30 \mathrm{~kg} \cdot \mathrm{m}^{-2}$ & 31.9 & 24.6 & 28.8 & 32.7 & 32.9 \\
\hline \multicolumn{6}{|c|}{ Number of exacerbations during baseline period } \\
\hline 0 & 88.5 & 82.1 & 70.5 & 95.8 & 96.6 \\
\hline Any ICS medication & 71.6 & 79.6 & 58.0 & 60.2 & 72.7 \\
\hline ICS/LABA or ICS+LABA & 30.8 & 47.1 & 43.8 & 41.8 & 51.0 \\
\hline ICS/LABA/LAMA or ICS/LABA+LAMA & 0.5 & 1.1 & 0.9 & 0.3 & 0.9 \\
\hline Any LTRA & 4.2 & 9.6 & 10.4 & 2.1 & 5.5 \\
\hline Any theophylline & 0.5 & 1.2 & 0.4 & 1.6 & 3.4 \\
\hline Other anti-allergic agents & 15.9 & 27.3 & 37.7 & 7.7 & 3.4 \\
\hline \multicolumn{6}{|l|}{ Asthma severity during baseline period } \\
\hline Mild (GINA steps 1-2) & 42.4 & 41.4 & 44.0 & 50.9 & 38.0 \\
\hline Moderate (GINA step 3) & 33.1 & 15.5 & 20.8 & 32.0 & 31.6 \\
\hline Severe (GINA steps 4-5) & 24.5 & 43.1 & 35.1 & 17.1 & 30.4 \\
\hline \multicolumn{6}{|l|}{ Comorbidities } \\
\hline Cardio-cerebrovascular disease & 5.4 & 8.3 & 5.0 & 9.0 & 2.5 \\
\hline
\end{tabular}

Data are presented as mean \pm SD or \%, unless otherwise stated. UK: United Kingdom; GPs: general physicians; BMI: body mass index; SABA: short-acting $\beta_{2}$-agonists; ICS: inhaled corticosteroids; LABA: long-acting $\beta_{2}$-agonists; LAMA: long-acting muscarinic antagonists; LTRA: leukotriene receptor antagonists; GINA: Global Initiative for Asthma. \#: calculated for adult patients only.

between 91 and 180 days, the average daily OCS dosage remained stable at 5.5-7.5 mg for patients who continued to meet the high-use definition in subsequent intervals through to 631-720 days (figure 5, supplementary table S1).

Across the four study countries, 3-7\% of patients with mild asthma at baseline became high OCS users during follow-up. Patients with severe asthma were more likely to become high OCS users than patients with mild or moderate disease (supplementary table S2). Prescription patterns and frequency of OCS use stratified by asthma severity and exacerbation history are presented in supplementary table S3. Patients with severe asthma at baseline had more OCS prescriptions and received greater average daily OCS dosages than patients with non-severe asthma. A similar trend was observed for patients with a history of exacerbations during the baseline period versus those without a history of exacerbations.

Characteristics of patients with high OCS use

Compared with low and non-OCS users, high OCS users were consistently more likely to be older, female, to have had more exacerbations and have greater asthma severity across the countries studied (supplementary table S4). In addition, high OCS users were more likely to have been prescribed ICS only, 


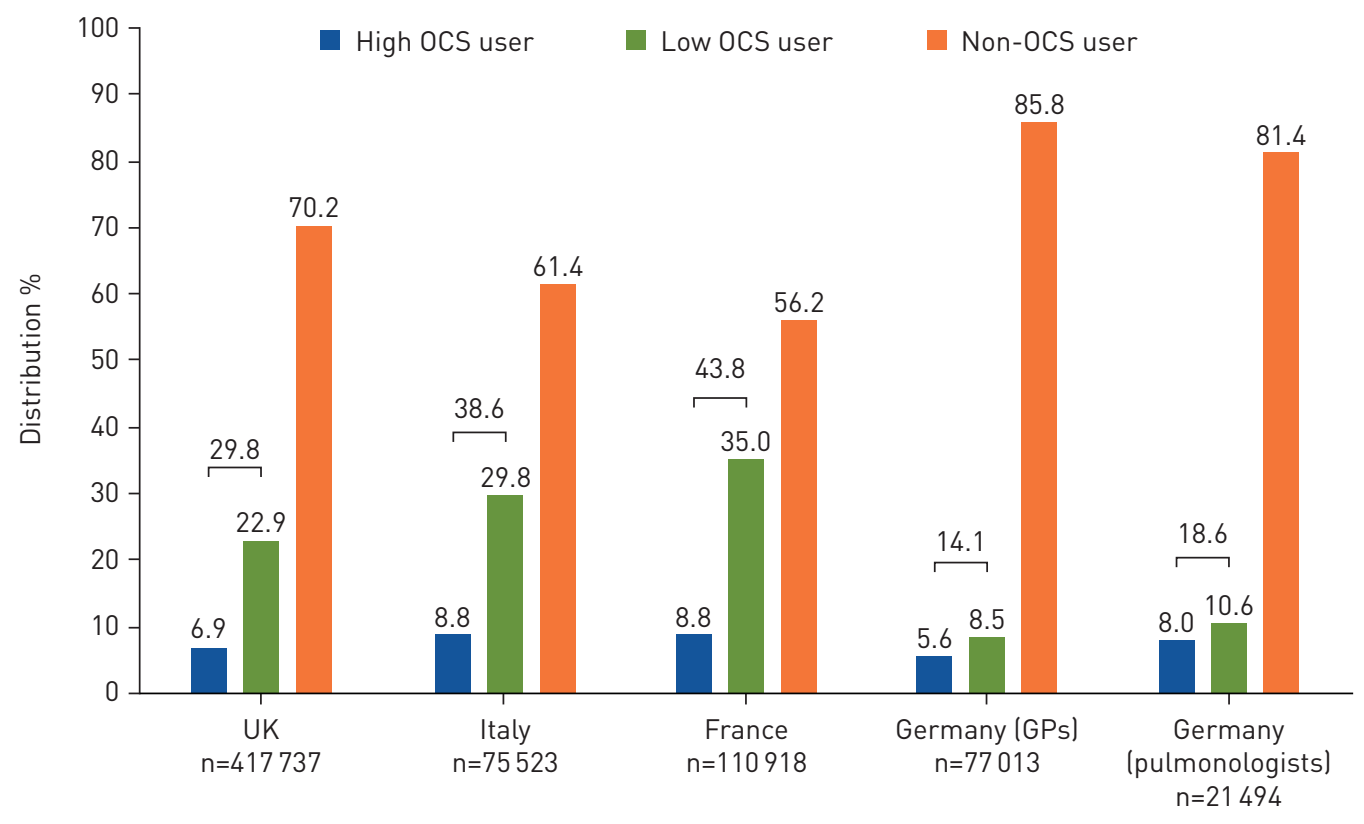

FIGURE 3 Distribution of oral corticosteroid (OCS) users during the study period. High OCS users are defined as having a cumulative dosage $\geqslant 450 \mathrm{mg}$ within 90 days (average daily OCS dosage $\geqslant 5 \mathrm{mg}$ ); low OCS users were prescribed OCS, but did not meet high OCS criteria; non-OCS users had no OCS prescription during the entire follow-up period. GPs: general physicians.

dual or triple therapy, leukotriene receptor antagonists, any theophylline, and other anti-allergic agents during the baseline period compared with low or non-OCS users.

\section{Discussion}

In this study of $>700000$ patients with active asthma, we aimed to describe the current state of OCS prescriptions for the treatment of asthma in France, Germany, Italy and the UK. In line with publications from database studies [23-25], we found that OCS prescribing was common (up to $44 \%$ of patients with asthma) across all countries studied. This finding corresponds with $21-50 \%$ of patients with asthma reporting steroid use in a large survey conducted for patients with asthma in Europe and Canada [26].

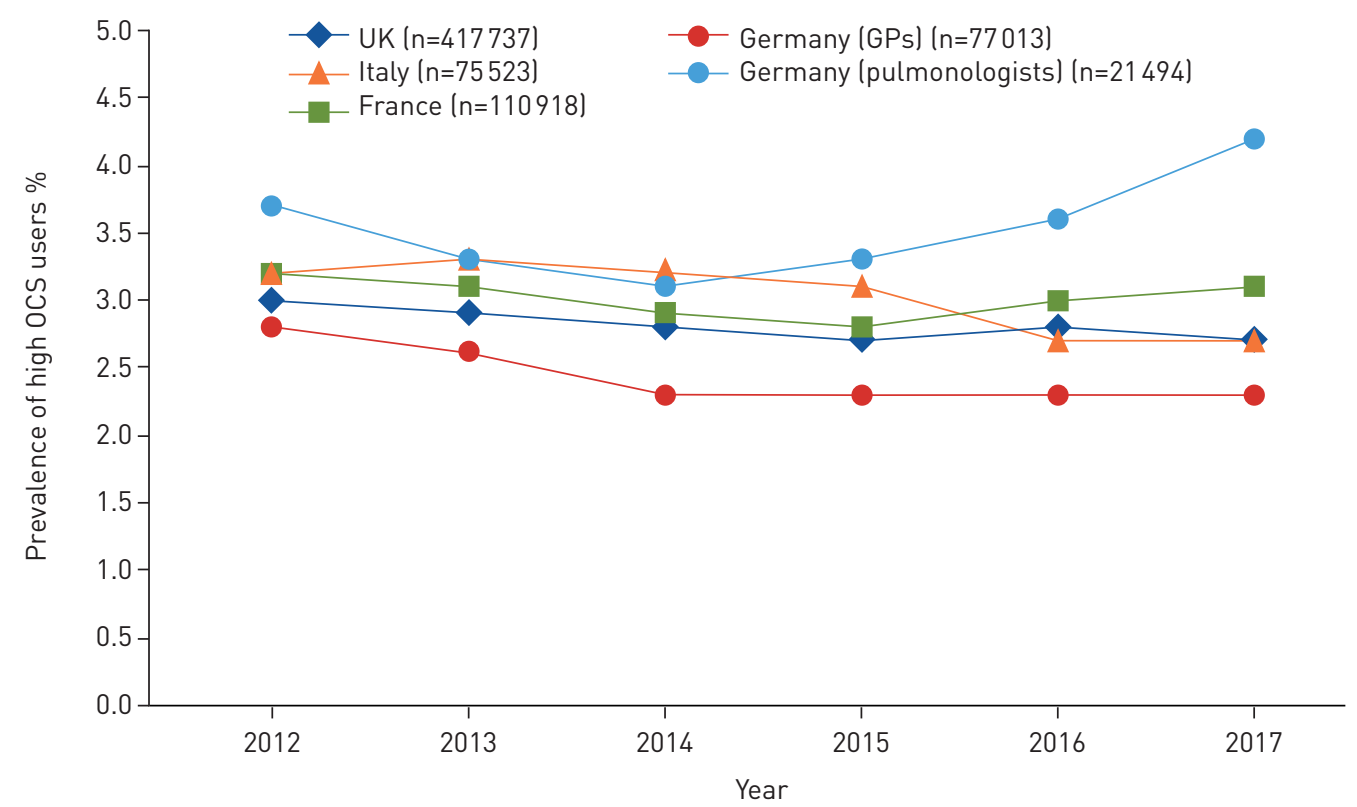

FIGURE 4 Percentage of patients with asthma identified as high oral corticosteroids (OCS) users by calendar year. UK: United Kingdom; GPs: general physicians. 
TABLE 2 Prescriptions and daily oral corticosteroid (OCS) dosage

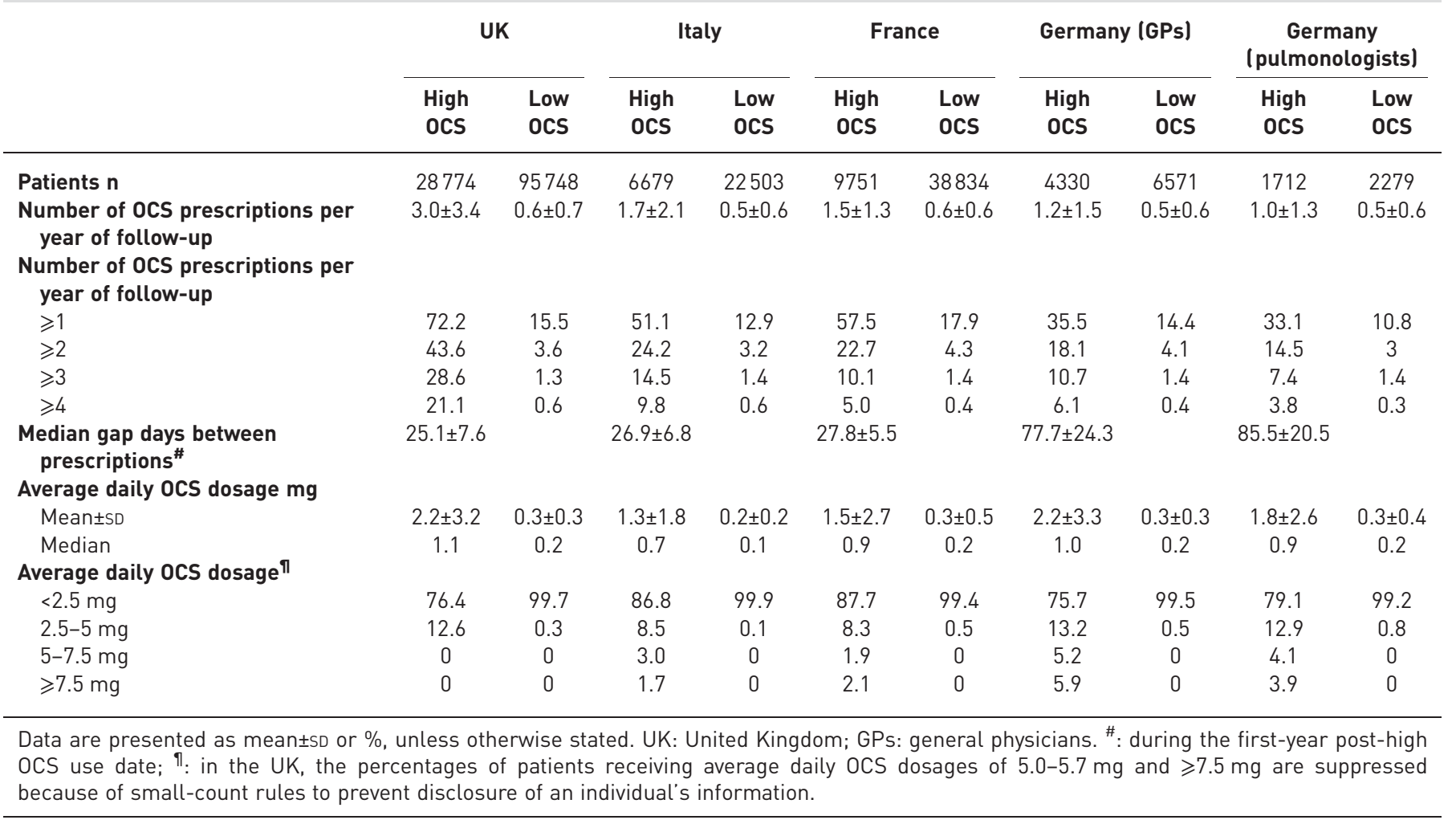

The variation in frequency of OCS prescriptions may have resulted from differences in prescribing practices across countries [27, 28], types of data used (e.g. administrative claims, electronic medical records, pharmacy prescriptions) and the definition of high OCS users (e.g. based on daily dosage, duration of prescription and number of refill prescriptions, as well as prescription cut-offs). Despite

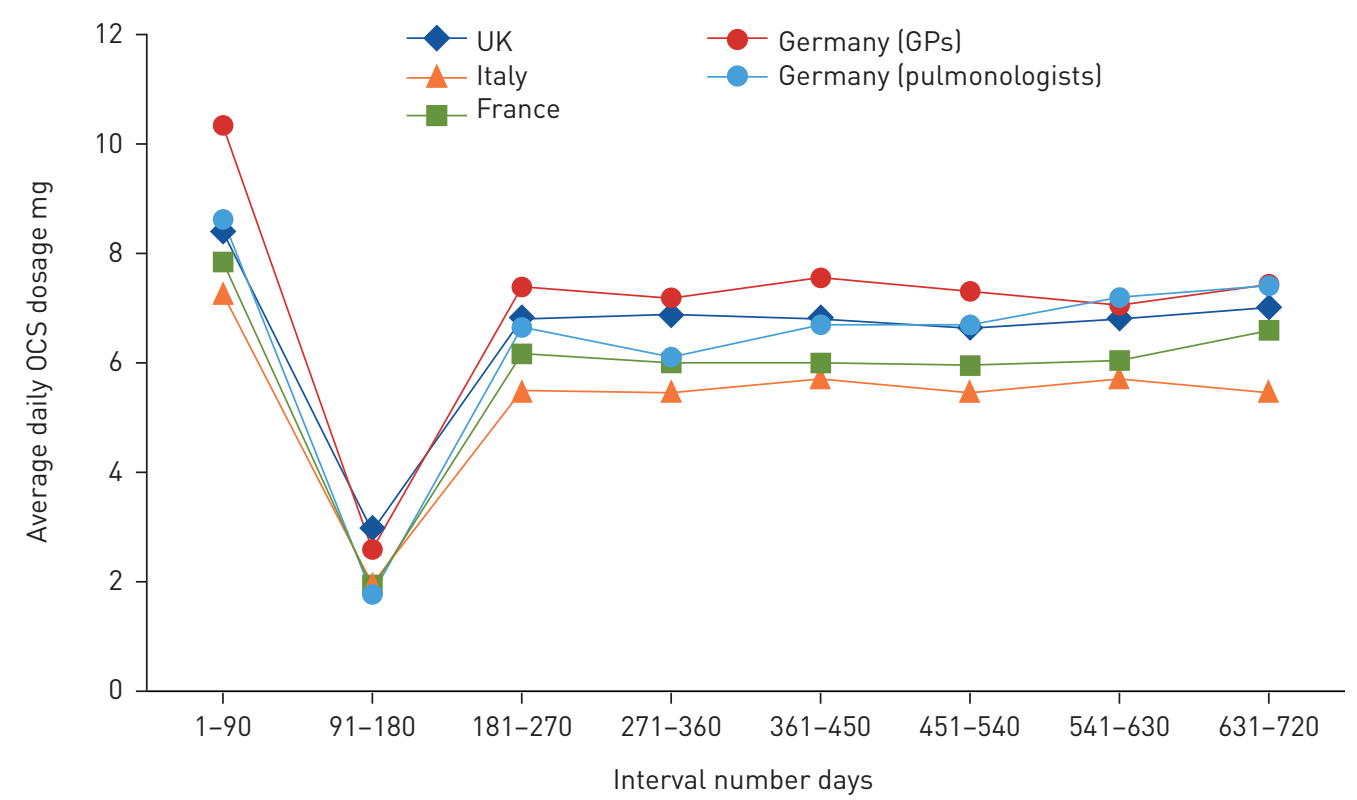

FIGURE 5 Average daily oral corticosteroid (OCS) dosage over time for patients identified as high OCS users. UK: United Kingdom; GPs: general physicians. Number of days is relative to high-user date (day 1). Patients are eligible for inclusion if they are identified as high OCS users at the beginning of or during the specific 90-day interval. 
variations in frequency of overall OCS prescription, the percentage of patients classified as high OCS users $(\geqslant 450 \mathrm{mg}$ in 90 days) at any time during the study follow-up remained stable at $6-9 \%$, and annual prevalence was stable at $\sim 3 \%$ across the included European countries. The annual prevalence of high OCS use observed in our study is less than the $8.2 \%$ prevalence of chronic OCS use reported in a similar study in the United States that defined chronic OCS use as $\geqslant 2.5 \mathrm{mg} \cdot$ day $^{-1}$ over 1 year [23]. It is noteworthy that for this study, we selected a high OCS use definition ( $\geqslant 450 \mathrm{mg}$ in 90 days) corresponding to an average daily OCS dosage $\geqslant 5 \mathrm{mg}$, which is known to be associated with an increased risk of OCS-related complications $[13,15,21-23]$.

Annual prevalence of high OCS use has remained stable since 2012, indicating little change in OCS prescribing patterns despite the recent introduction of OCS-sparing therapies. Additionally, it is possible that prescribing patterns shifted from maintenance OCS to more short-term therapy or vice versa, which may have resulted in stable prevalence of high OCS use according to our study definition. Although a robust distinction between maintenance and short-term therapy was beyond the scope of this study, it is possible that other methods could have made this distinction.

Although few patients in the UK were prescribed an average daily OCS dosage $>5 \mathrm{mg}$, a greater percentage of UK patients received three or more OCS prescriptions per year compared with other countries. Seemingly, UK patients were prescribed lower dosages across more prescriptions compared with the other countries in this study. In contrast, Germany had the least number of prescriptions but similar average daily dosages compared with the other countries, suggesting fewer prescriptions with larger dosages per prescription than other countries. This probably reflects differences in the healthcare systems and reimbursement practices between the studied countries. A longitudinal UK study found that incidence of adverse outcomes of systemic corticosteroid use increased with cumulative OCS exposure, starting at a cumulative annual dosage as low as $0.5 \mathrm{~g}$ [15]. In our study, overall OCS exposure for both high and low OCS users was relatively consistent across all countries. The average daily dosage of $\sim 1.5-2 \mathrm{mg}$ for high OCS users is equivalent to a cumulative annual OCS dosage of $0.55-0.73 \mathrm{~g}$, suggesting that high OCS users with asthma may be at high risk of OCS-related adverse effects. In all countries, patients who continued to meet the definition of high OCS use had a daily exposure of 5.5-7.5 mg, equivalent to cumulative annual dosages of 2-2.7 g. It is unfortunate that a group of patients continued to be exposed to a stable and significant degree of OCS for a prolonged period (up to 2 years) (figure 5), placing them at particularly high risk of adverse effects during this period and possibly beyond. The lesser daily OCS dosage during the second 90-day interval (i.e. days 91-180) was the result of including all patients who met the high-use criterion in the previous (i.e. the first 90-day interval) or current interval in the denominator, while a percentage of the former decreased OCS use during the second 90-day interval.

In this study, OCS users, particularly high OCS users, were, in general, consistently older and more often female across countries compared with non-OCS users. In addition, high OCS users often had more severe asthma (GINA steps 4-5) and more baseline exacerbations compared with low OCS users. This was to be expected considering the recommendations for prescribing maintenance OCS to patients with severe, uncontrolled asthma, which would more likely result in patients meeting the high-use definition compared with patients with milder asthma for which OCS would most likely be prescribed for occasional exacerbations [23, 29]. Although this study did not address the association between OCS use and OCS-related comorbidities, the percentage of high OCS users with comorbidities was greater than the percentage of low OCS users with comorbidities, confirming the knowledge that OCS use is associated with significant comorbidities for patients [11-15].

The main strengths of the current study are the inclusion of a large number of patients with asthma from multiple data sources across different European countries and the use of standard definitions and algorithms for OCS exposure, disease severity and clinical outcomes. The selected data sources are representative samples of the national populations of each country examined, and the data collected should reflect routine clinical practices in each country. Despite differences in asthma treatment practices [27, 28, 30], reimbursement guidelines, treatment or referral incentives, national healthcare practices [31] and health delivery systems [32], we found consistent patterns of OCS prescriptions across the included countries, which adds confidence to our findings. As with many similar studies, having a recorded prescription does not mean the patient took the medication. Therefore, we could have overestimated OCS exposure. In addition, OCS exposure could have been overestimated because OCS prescriptions were not recorded with the medical condition being treated. To mitigate this risk, we excluded patients with several diseases that are commonly treated with OCS. Nor could we account for stockpiling of medication, which could have led to underestimation of exacerbations. Furthermore, with the lack of a consensus definition for high OCS use in the scientific community, it is difficult to compare our findings with those from studies with other definitions. Alternative approaches to defining high OCS use include using the number of OCS prescriptions within a specific period or OCS use duration. The primary care databases did not 
contain information on biologic therapies, which limited the possibility of describing OCS use in the context of OCS-sparing therapies.

In summary, we found that OCS prescriptions for asthma management are common in France, Germany, Italy and the UK. This study highlights that a proportion of patients with asthma are exposed to high daily OCS dosages over a long period of time, and a smaller number of patients with mild disease are high OCS users. Taken together, these findings suggest suboptimal asthma management in all study countries. Further research is needed to understand the reasons for continued OCS prescribing, despite the evolving knowledge in this field and the availability of alternative OCS-sparing therapies. Considering the 2019 GINA guidelines, these findings provide a European benchmark for future reduction of OCS prescriptions in asthma management.

Acknowledgements: Medical writing was provided by Yukti Singh from IQVIA (Gurugram, India) for early drafts and by JK Associates, Inc. (Conshohocken, PA, USA), and Michael A. Nissen of AstraZeneca (Gaithersburg, MD, USA) for later and final drafts. Writing support was funded by AstraZeneca. The results described in this article were presented in part at the European Academy of Allergy and Clinical Immunology (EAACI) and European Respiratory Society (ERS) 2019 Annual Meetings. IQVIA Medical Research Data (IMRD) incorporates data from The Health Improvement Network (THIN). THIN is a registered trademark of Cegedim SA in the UK and other countries. Reference made to the THIN database is intended to be descriptive of the data asset licensed by IQVIA. This work uses de-identified data provided by patients as a part of their routine primary care.

Support statement: Funding for this study was provided by AstraZeneca. Funding information for this article has been deposited with the Crossref Funder Registry.

Conflict of interest: T.N. Tran is an employee of AstraZeneca. E. King was external consultant with IQVIA at the time these analyses were conducted. R. Sarkar was an employee of IQVIA at the time these analyses were conducted. C. Nan is an employee of AstraZeneca and shareholder in GlaxoSmithKline. A. Rubino was an Evidera employee at the time these analyses were conducted, and is now an AstraZeneca employee. C. O'Leary is an employee of IQVIA. R. Muzwidzwa is an employee of IQVIA. L. Belton is contractor for AstraZeneca Cambridge; Imperial College received consulting fees from AstraZeneca. J.K. Quint is an employee of Imperial College London; IQVIA received payment from AstraZeneca for the conduct of this study.

\section{References}

1 Global Asthma Network. The Global Asthma Report 2018. www.globalasthmareport.org/. Date last accessed: November 2019.

2 Gibson GJ, Loddenkemper R, Lundbäck B, et al. Respiratory health and disease in Europe: the new European Lung White Book. Eur Respir J 2013; 42: 559-563.

3 de Marco R, Cappa V, Accordini S, et al. Trends in the prevalence of asthma and allergic rhinitis in Italy between 1991 and 2010. Eur Respir J 2012; 39: 883-892.

4 Gößwald A, Lange M, Kamtsiuris $\mathrm{P}$, et al. Bundesweite Quer- und Längsschnittstudie im Rahmen des Gesundheitsmonitorings des Robert Koch-Instituts. [DEGS: German Health Interview and Examination Survey for Adults. A nationwide cross-sectional and longitudinal study within the framework of health monitoring conducted by the Robert Koch Institute]. Bundesgesundheitsblatt Gesundheitsforschung Gesundheitsschutz 2012; 55: 775-780.

5 Jarvis D, Newson R, Lotvall J, et al. Asthma in adults and its association with chronic rhinosinusitis: the GA ${ }^{2}$ LEN survey in Europe. Allergy 2012; 67: 91-98.

6 Langen U, Schmitz R, Steppuhn H. Ergebnisse der Studie zur Gesundheit Erwachsener in Deutschland (DEGS1). [Prevalence of allergic diseases in Germany: results of the German Health Interview and Examination Survey for Adults (DEGS1)]. Bundesgesundheitsblatt Gesundheitsforschung Gesundheitsschutz 2013; 56: 698-706.

7 Mukherjee M, Stoddart A, Gupta RP, et al. The epidemiology, healthcare and societal burden and costs of asthma in the UK and its member nations: analyses of standalone and linked national databases. BMC Med 2016; 14: 113.

8 To T, Stanojevic S, Moores G, et al. Global asthma prevalence in adults: findings from the cross-sectional world health survey. BMC Public Health 2012; 12: 204.

9 Global Initiative for Asthma. Pocket Guide for Asthma Management and Prevention 2019. https://ginasthma.org/ pocket-guide-for-asthma-management-and-prevention/ Date last accessed: November 2019.

10 BTS/SIGN. British Guideline for the Management of Asthma. SIGN 158. 2019. www.brit-thoracic.org.uk/ quality-improvement/guidelines/asthma/ Date last accessed: November 2019.

11 Bleecker ER, Menzies-Gow AN, Price DB, et al. Systematic literature review of systemic corticosteroid use for asthma management. Am J Respir Crit Care Med 2020; 201: 276-293.

12 Waljee AK, Rogers MA, Lin P, et al. Short term use of oral corticosteroids and related harms among adults in the United States: population based cohort study. BMJ 2017; 357: j1415.

13 Sullivan PW, Ghushchyan VH, Globe G, et al. Oral corticosteroid exposure and adverse effects in asthmatic patients. J Allergy Clin Immunol 2018; 141: 110-116.

14 Bloechliger M, Reinau D, Spoendlin J, et al. Adverse events profile of oral corticosteroids among asthma patients in the UK: cohort study with a nested case-control analysis. Respir Res 2018; 19: 75.

15 Price DB, Trudo F, Voorham J, et al. Adverse outcomes from initiation of systemic corticosteroids for asthma: long-term observational study. J Asthma Allergy 2018; 11: 193-204.

16 Denburg MR, Haynes K, Shults J, et al. Validation of The Health Improvement Network (THIN) database for epidemiologic studies of chronic kidney disease. Pharmacoepidemiol Drug Saf 2011; 20: 1138-1149.

17 Lewis JD, Schinnar R, Bilker WB, et al. Validation studies of The Health Improvement Network (THIN) database for pharmacoepidemiology research. Pharmacoepidemiol Drug Saf 2007; 16: 393-401. 
18 Becher H, Kostev K, Schröder-Bernhardi D. Validity and representativeness of the 'Disease Analyzer' patient database for use in pharmacoepidemiological and pharmacoeconomic studies. Int J Clin Pharmacol Ther 2009; 47: $617-626$.

19 Jouaville SL, Miotti H, Coffin G, et al. Validity and limitations of the Longitudinal Patient Database France for use in pharmacoepidemiological and pharmacoeconomics studies. Value Health 2015; 18: A18.

20 Istituto Di Ricerca Della SIMG. IX Report Health SearchDate last accessed: February 10, 2020. https:// healthsearch.it/documenti/Archivio/Report/2016/completo.pdf.

21 Al Efraij K, Johnson KM, Wiebe D, et al. A systematic review of the adverse events and economic impact associated with oral corticosteroids in asthma. J Asthma 2019; 56: 1334-1346.

22 Dalal AA, Duh MS, Gozalo L, et al. Dose-response relationship between long-term systemic corticosteroid use and related complications in patients with severe asthma. J Manag Care Spec Pharm 2016; 22: 833-847.

23 Zeiger RS, Schatz M, Li Q, et al. Burden of chronic oral corticosteroid use by adults with persistent asthma. J Allergy Clin Immunol Pract 2017; 5: 1050-1060.

24 Price D, Kaplan A, Jones R, et al. Long-acting muscarinic antagonist use in adults with asthma: real-life prescribing and outcomes of add-on therapy with tiotropium bromide. J Asthma Allergy 2015; 8: 1-13.

25 Ekström M, Nwaru BI, Hasvold P, et al. Oral corticosteroid use, morbidity and mortality in asthma: a nationwide prospective cohort study in Sweden. Allergy 2019; 74: 2181-2190.

26 Sastre J, Fabbri LM, Price D, et al. Insights, attitudes, and perceptions about asthma and its treatment: a multinational survey of patients from Europe and Canada. World Allergy Organ J 2016; 9: 13.

27 Lagerløv P, Veninga CC, Muskova M, et al. Asthma management in five European countries: doctors' knowledge, attitudes and prescribing behaviour. Drug Education Project (DEP) group. Eur Respir J 2000; 15: 25-29.

28 Wahlström R, Hummers-Pradier E, Lundborg CS, et al. Variations in asthma treatment in five European countries - judgement analysis of case simulations. Fam Pract 2002; 19: 452-460.

29 Fardet L, Petersen I, Nazareth I. Description des prescriptions de corticothérapies orales en population générale. [Description of oral glucocorticoid prescriptions in general population]. Rev Med Interne 2011; 32: 594-599.

30 Janson C, Chinn S, Jarvis D, et al. Physician-diagnosed asthma and drug utilization in the European Community Respiratory Health Survey. Eur Respir J 1997; 10: 1795-1802.

31 McCarthy M. Sustainable general practice: looking across Europe. Br J Gen Pract 2016; 66: 36.

32 Nolte E, Knai C. Approaches to chronic disease management in Europe. In: Nolte E, Knai C, Saltman RB, eds. European Observatory on Health Systems and Policies Observatory Studies Series, No 37. Copenhagen, World Health Organization, 2014. 